\title{
Magnetic mind games
}

\author{
Using short magnetic \\ pulses, neuroscientists \\ are reaching into the \\ human skull and
} temporarily altering volunteers' brain activity. Marina Chicurel takes an induction course.

0 tudying the human brain can be a frustrating business. Although sophisticated imaging techniques can offer snapshots of activity, direct intervention in the brains of humans is ethically off-limits. It is no wonder that neuroscientists sometimes feel like visitors to a museum — they can look but not touch.

But things are beginning to change. Neuroscientists studying human cognitive processes are now making tentative use of a technique that can temporarily alter their subjects' brain activity. Insights into everything from language to conscious awareness are emerging, as well as hints that the technique could help treat some mental disorders. "There are just some extraordinary things coming out," says Michael Gazzaniga of Dartmouth College in Hanover, New Hampshire, editor-in-chief of the Journal of Cognitive Neuroscience.

Before technology for imaging the human brain emerged, neuroscientists interested in cognitive functions were mostly limited to studying patients with brain lesions - damage to specific areas of the brain. By looking for links between the patients' symptoms and the site of the damage, researchers pinpointed brain areas involved in abilities such as language,

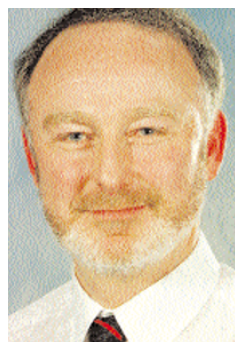

Anthony Barker used magnets to control movement.

\section{learning and memory.}

During the 1980s and 1990s, techniques such as functional magnetic resonance imaging (fMRI), which tracks brain activity by monitoring blood flow, allowed neuroscientists to see which areas of the brain were active during specific tasks. A flood of information about the functions of

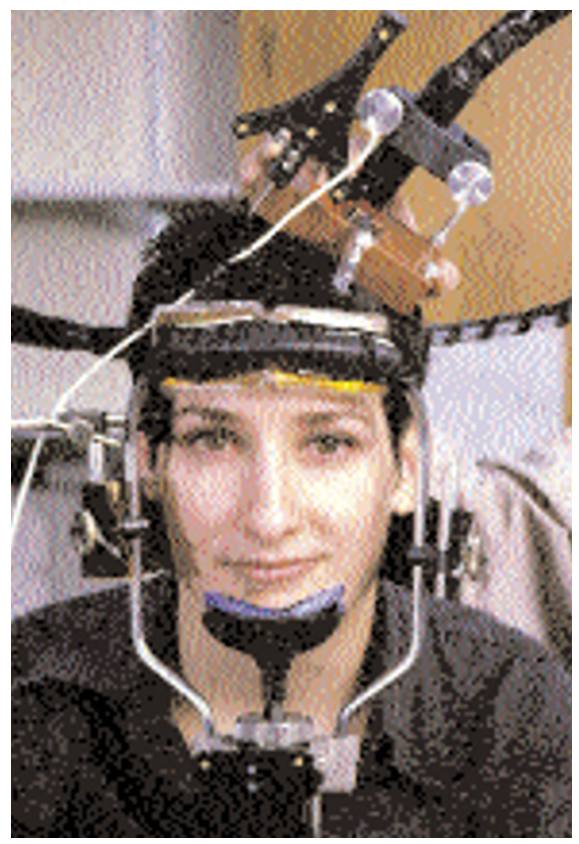

different brain areas followed.

But such techniques have their limits. “There's no causality in it," complains Tomáš Paus, a neuroscientist at McGill University in Montreal, Canada. "You can never say whether one brain region is influencing another, or whether they are active together because some third region is driving both." Rather than just passively observing the brain, researchers would like to manipulate it directly.

\section{Polar explorers}

Scientists had long suspected that magnetic fields might allow them to do this. Brain cells send electrical signals along the fibres that make up their communication networks. Because changing magnetic fields can induce current in electrical conductors, researchers thought that a magnetic pulse might stimulate currents in brain cells, and so alter brain activity. Early attempts to do this date back to the nineteenth century, but it wasn't until 1985 that devices capable of producing the short, intense pulses needed to stimulate the brain were developed.

The breakthrough was made by Anthony Barker, a medical physicist at the Royal Hallamshire Hospital in Sheffield, UK. Barker used a 2-tesla magnetic pulse about 1 millisecond long to stimulate the brain area that controls finger movement. Unintentionally, the volunteer's fingers twitched ${ }^{1}$. "It caused a good deal of excitement," recalls Barker.

The technique, known as transcranial magnetic stimulation (TMS), remains similar today. There are hints that the intensity and frequency of the induced current can influence whether the stimulation increases or damps down brain activity ${ }^{2,3}$. But TMS cannot, at present, exert precise control over the brain. It puts in "random neural noise", explains Vincent Walsh of the University of

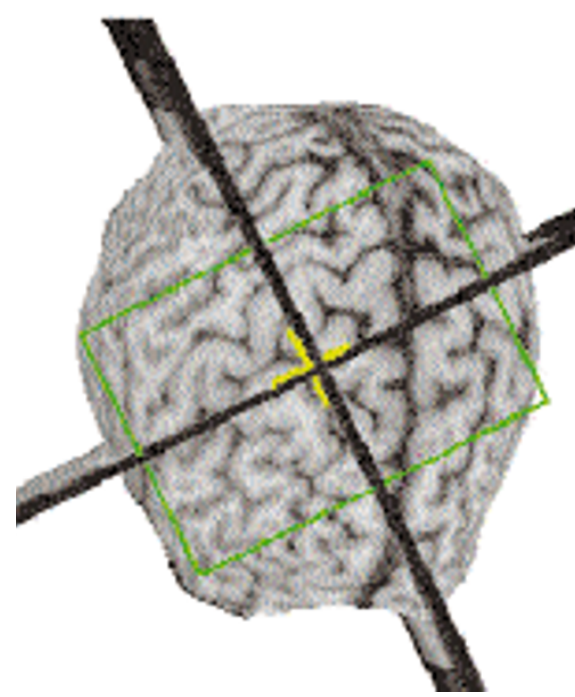

A century-old dream to influence brain activity with magnets (below), is now a reality using powerful coils (left) and imaging (above).

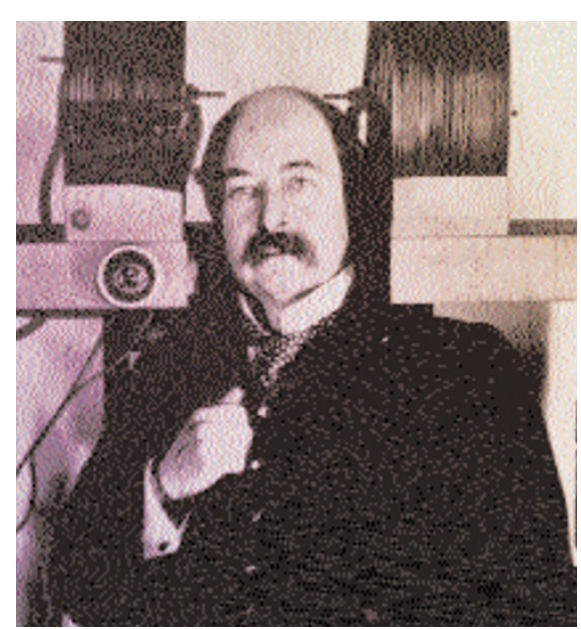

Oxford, UK, who has used TMS to study cognitive functions such as visual awareness.

Single pulses affect brain function for just a few milliseconds. But a train of pulses, typically delivered at rates of about one per second, can disrupt brain activity for tens of minutes. This technique, known as repetitive TMS (rTMS), creates 'virtual' lesions for neuroscientists to experiment on.

Last year, for example, Alfonso Caramazza and colleagues at Harvard University used rTMS to reveal how our brains seem to use different regions to handle verbs and nouns. Caramazza was studying the left prefrontal cortex, an area thought to be involved in the ability to conjugate verbs. Previous lesion studies had proved inconclusive, partly because the damage often extended beyond the area of interest. And, as with other studies of brain damage, it was impossible to gauge the extent to which other parts of the patients' brains had compensated for the damage.

"With brain-damaged patients we're at the mercy of nature," says Caramazza. “That's why I became a TMS convert.” When Caramazza applied rTMS to the left prefrontal cortex he found that his subjects had difficulty conju- 


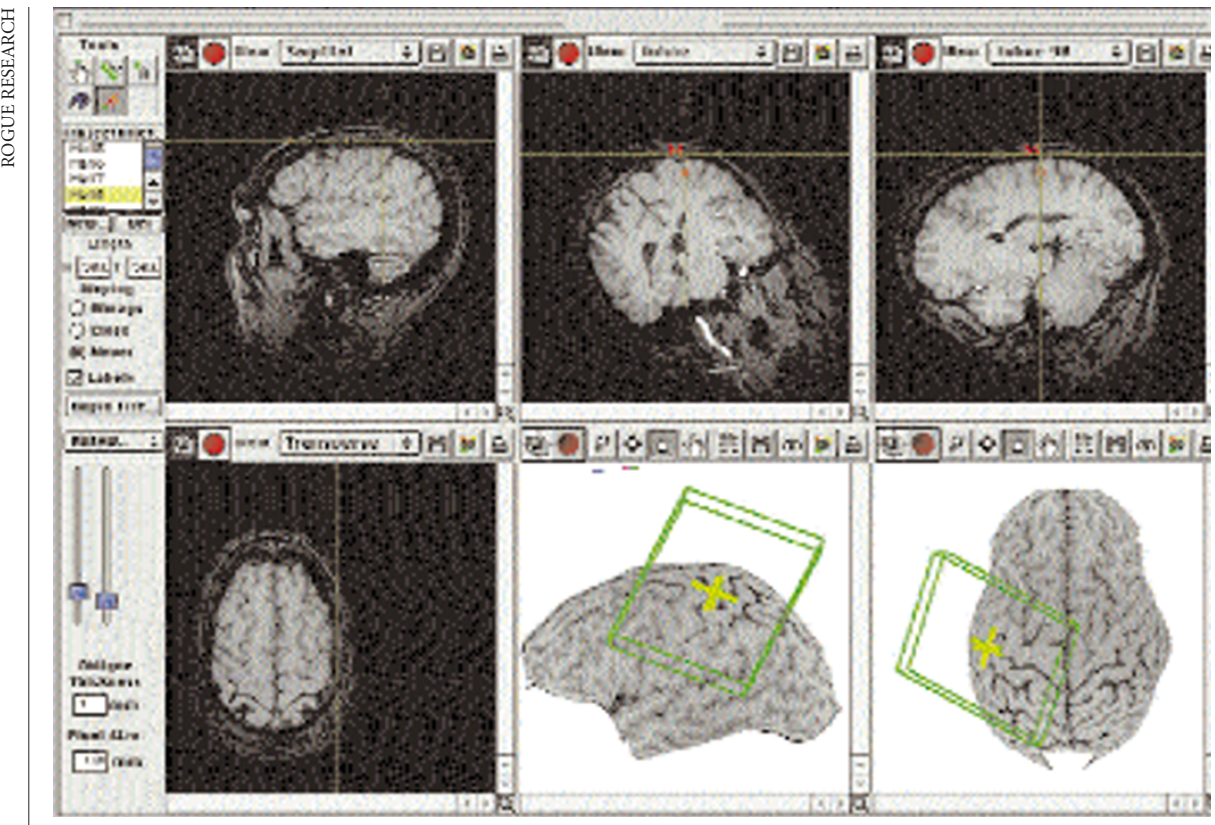

Precise picture: imaging techniques allow the magnetic stimulus to be applied in a specific position.

of the National Institute of Neurological Disorders and Stroke in Bethesda, Maryland, thinks that these evaluations should be standardized to ensure that they pick up subtle effects. "You might want to have people stay longer, you might want to have additional testing," he says. "There have been very few studies considering this."

Despite these questions, most agree that isolated sessions of rTMS, such as those experienced by volunteers in cognitive studies, do not induce permanent changes. But repeated bouts of rTMS can have long-lived effects, and some hope to harness them to treat mental disorders. In 1995, for example, after noticing that depressed patients had low levels of activity in their left prefrontal cortices, Mark George, a psychiatrist at the Medical University of South Carolina in Charleston, tried boosting activity in that region using $\mathrm{rTMS}^{13}$. George says that his patients improved, but similar studies conducted since have proved to be less clear-cut.

A team led by Tal Burt, a psychiatrist at Columbia University in New York, has

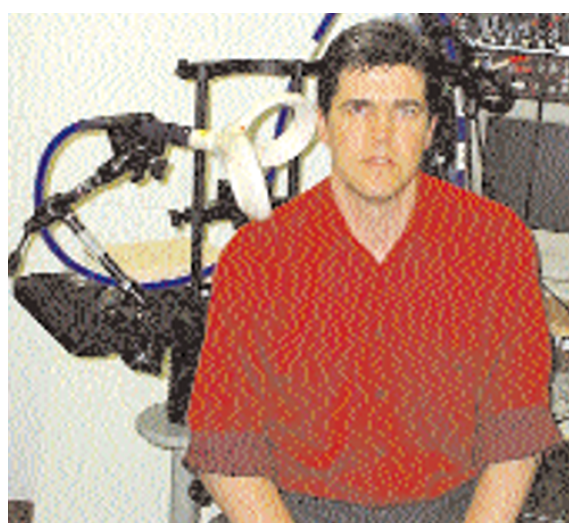

Vincent Walsh believes that there are many easy experiments involving TMS waiting to be done. recently collated the results of 25 such studies $^{14}$. The group concludes that rTMS has a modest antidepressant effect, of uncertain clinical value, but which could improve as researchers refine their methods. Factors such as coil placement, stimulation frequency and the influence of patient variability now need to be investigated. George is planning largescale trials. "The questions now are: how big is that effect, can it be made to last, is it clinically useful, what are the use parameters that maximize the effect?" he says.

\section{Virtual surgery}

Other disorders are also being tackled with rTMS. Last year, Massimiliano Oliveri, a neuroscientist at the University of Palermo in Italy, working with colleagues at the University of Turin, showed how the technique can be used to treat hemispatial neglect, a condition in which patients have difficulty paying attention to objects on one side of their visual field.

The condition is often caused by damage to parts of the brain called the parietal cortex. Under normal circumstances, the left and right sides of this area inhibit each other. But when one side is damaged, the other becomes hyperactive. Although, to a degree, the brain may compensate for this, Oliveri reasoned that he might be able to balance the activity by creating a virtual lesion in the undamaged side. Initial results were promising, with the patients showing improved awareness during stimulation $^{15}$, and Oliveri is now using stimuli designed to produce longer-lived changes $-1 \mathrm{~Hz}$ for 10 minutes every day for five days. The eight patients he has treated, and followed for a month so far, have improved $40 \%$ faster than non-treated controls, he says.

TMS may also be able to go one step further, and enhance normal peoples' thought processes. Last year, Grafman applied rTMS to normal volunteers while they solved a reasoning puzzle ${ }^{16}$. "We decided to see if we could actually make people faster," he says. By applying rTMS to the prefrontal cortex, an area that is active when people solve certain types of visual puzzle, Grafman reduced the time taken by subjects to solve a problem involving geometric shapes.

But it is unclear why such experiments work. Walsh says that noise can boost performance when it is used to block an inhibiting area, such as in Oliveri's studies of neglect, but it is difficult to see how it could enhance a circuit's precisely tuned pattern of activity. Grafman acknowledges this apparent paradox, but points out that noise can, in some systems, aid a change from one state to another - a phenomenon known as stochastic resonance.

Some scientists are uneasy about this area of research. Pascual-Leone believes it might be possible to use TMS to enhance a wealth of mental skills. "But those experiments shouldn't be done without a very serious debate," he says. "And who is to decide what behaviour should be modified, and by whom, and for what purpose?"

Others do not see an ethical dilemma in such experiments. George has been funded by the US Department of Defense to study whether TMS can help to improve memory. He argues that such methods are no different from accepted means of boosting performance. "We do things all the time to try to enhance our performance," says George. "We exercise, we practice. The idea of using a physical stimulation to do that doesn't seem to me to broach any new ground.'

Whether or not such controversial projects succeed, TMS enthusiasts see a bright future for the technique. After more than a decade of relative obscurity, they say TMS is beginning to take its place in the toolboxes of cognitive neuroscientists. "People have started realizing how many unique opportunities magnetic stimulation offers," says PascualLeone. "I think they've started to get it." Marina Chicurel is a writer in Santa Cruz, California.

1. Barker, A. T., Jalinous, R. \& Freeston, I. L. Lancet 1,

1106-1107 (1985)

2. Chen, R. et al. Neurology 48, 1398-1403 (1997).

3. Maeda, F., Keenan, J. P., Tormos, J. M., Topka, H. \&

Pascual-Leone, A. Clin. Neurophysiol. 111, 800-805 (2000).

4. Shapiro, K. A., Pascual-Leone, A., Mottaghy, F. M., Gangitano,

M. \& Caramazza, A. J. Cogn. Neurosci. 13, 713-720 (2001).

5. Mellet, E., Petit, L., Mazoyer, B., Denis, M. \& Tzourio, N.

Neuroimage 8, 129-139 (1998).

6. Kosslyn, S. M. et al. Science 284, 167-170 (1999).

7. Fierro, B. et al. NeuroReport 11, 1519-1521 (2000).

8. Rossi, S. et al. Nature Neurosci. 4, 948-952 (2001).

9. Keenan, J. P., Nelson, A., O'Connor, M. \& Pascual-Leone, A.

Nature 409, 305 (2001).

10. Harmer, C. J., Thilo, K. V., Rothwell, J. C. \& Goodwin, G. M. Nature Neurosci. 4, 17-18 (2001)

11. Pascual-Leone, A. \& Walsh, V. Science 292, 510-512 (2001). 12. Wassermann, E. M. Electroencephalogr. Clin. Neurophysiol. 108, 1-16 (1998).

13. George, M. S. et al. NeuroReport 6, 1853-1856 (1995).

14. Burt, T., Lisanby, S. H. \& Sackeim, H. A. Int. J.

Neuropsychopharmacol. (in the press).

15. Oliveri, M. et al. Neurology 57, 1338-1340 (2001). 16. Boroojerdi, B. et al. Neurology 56, 526-528 (2001). 